\title{
Serological evidence of Legionella species infection in acute exacerbation of COPD
}

\author{
D. Lieberman*,\#, D. Lieberman\#, O. Shmarkov\#, Y. Gelfer\#, M. Ben-Yaakov", Z. Lazarovich", \\ I. Boldur
}

Serological evidence of Legionella species infection in acute exacerbation of COPD. D. Lieberman, D. Lieberman, O. Shmarkov, Y. Gelfer, M. Ben-Yaakov, Z. Lazarovich, I. Boldur. C) ERS Journals Ltd 2002.

ABSTRACT: A prospective study was conducted to identify and characterize hospitalizations for acute exacerbations of chronic obstructive pulmonary disease (AECOPD) with serological evidence of infection with Legionella spp. (Lsp).

Two-hundred and forty hospital admissions for AECOPD of 213 patients were included in the study. Paired sera were obtained for each of the admissions and were tested for 41 different serogroups of Lsp, using microimmunofluorescence-serology. Only a significant change in immunoglobulin- $G$ and/or immunoglobulin-M antibody titres was considered diagnostic.

In 40 admissions $(16.7 \%)$ there was serological evidence of infection with Lsp (LspH). Legionella pneumophila 1 was identified in nine admissions, L. pneumophila $3-15$ in 19 and nonpneumophila in 22 . In $26 \mathrm{LspH}(65 \%)$ there was serological evidence of infection with at least one other respiratory pathogen. Compared to the 200 admissions without Lsp $(\mathrm{NLspH})$, the $\mathrm{LspH}$ patients were younger $(\mathrm{p}<0.05)$ and more hypoxaemic $(\mathbf{p}<\mathbf{0 . 0 4})$. None of the cases in the LspH group had an abrupt onset of disease, compared to $58(29.0 \%)$ in the $\mathrm{NLspH}$ group $(p<0.0001)$. The incidence of myalgia/arthralgia was $55 \%$ for $\mathrm{LspH}$ compared to $37 \%$ for $\mathrm{NLspH}(\mathrm{p}<0.03)$.

To conclude, serological evidence of infection with Legionella spp. is common among patients hospitalized with acute exacerbations of chronic obstructive pulmonary disease. In most hospital admissions with serological evidence of infection with Legionella spp. an additional respiratory pathogen can be identified. Acute exacerbation develops gradually in these patients and is characterized clinically by more systemic manifestations than hospital admissions without serological evidence of infection with Legionella spp. The true interpretation and practical relevance of these findings should be determined in further studies.

Eur Respir J 2002; 19: 392-397.

\begin{abstract}
*The Pulmonary Unit and ${ }^{\#}$ Division of Internal Medicine, Soroka Medical Centre and the Faculty of Health Sciences, Ben-Gurion University of the Negev, Beer-Sheva, and the Microbiology Laboratory, Asaf Harofeh Medical Center, Zerifin, Bar-Ilan University, Ramat-Gan, Israel.
\end{abstract}

\author{
Correspondence: D. Lieberman \\ Pulmonary Unit \\ Soroka Medical Centre \\ Beer-Sheva \\ Israel 84101 \\ Fax: 97286403022 \\ E-mail: Lieberma@bgumail.bgu.ac.il
}

Keywords: Chronic obstructive pulmonary disease exacerbation

Legionella

Received: June 282001

Accepted after revision October 12001
Acute exacerbation (AE) is common in the course of chronic obstructive pulmonary disease (COPD). It has a substantial detrimental effect on patients' quality of life and increases utilization of health care services. The pathophysiological basis of $\mathrm{AE}$ is usually infectious. The frequency distribution of the various pathogens causing acute exacerbations of chronic obstructive pulmonary disease (AECOPD), as reported in different studies, depends to a large degree on the methodology and particularly on the diagnostic methods used to identify the specific infectious agents.

Legionella spp. (Lsp), which are an important cause of community-acquired pneumonia (CAP) [1], are usually almost totally absent from similar lists for AECOPD. The only context in which infection with Lsp is mentioned in COPD patients relates to the causes of CAP in this population [2], in which they are identified as the cause in $9 \%$ of the cases [3]. Recently,
Lsp was identified as the cause of AE in $6 \%$ of cases of chronic bronchitis [4].

In the framework of a comprehensive study of the infectious causes in patients hospitalized with AECOPD, the authors collected data on a large number of hospital admissions and a broad range of infective causes, using sophisticated and sensitive diagnostic techniques. The frequency distribution of all infective agents identified has been published elsewhere [5]. The objective of the present paper was to report findings that relate specifically to the group of AECOPD hospital admissions in which Lsp was identified as an aetiological factor (LspH). It was considered particularly important to describe the frequency distribution of the various Legionella serogroups and the other infective agents identified in these admissions, and to compare demographical, clinical and therapeutic characteristics, as well as outcomes in the LspH group, with similar variables in 
hospital admissions in which Lsp was not identified $(\mathrm{NLspH})$.

\section{Patients and methods}

\section{Patients}

All hospital admisssions for AECOPD during the period between November 1, 1997-March 15, 1999 in the internal medicine and intensive care wards of the Soroka Medical Centre in Beer-Sheva, Israel, that met the inclusion criteria and where patients gave consent to participate, were included in the study. All first admissions in the study period were included as well as repeat admissions for AECOPD of patients in the study population, if this occurred at least 6 months after the initial admission for which the patient was recruited into the study. No more than one repeat admission was included in the study data for any particular patient.

Inclusion criteria were all of the following: 1) age $>40 \mathrm{yrs}$; 2) chronic airway obstruction as determined by spirometry (performed 6 months prior to hospitalization or within 1-2 months following it) with a forced expiratory volume in one second (FEV1) value $<70 \%$ of expected and an FEV1/forced vital capacity $(\mathrm{FVC})$ ratio $<0.7 ; 3$ ) a smoking history of at least 20 pack-yrs; and 4) in the week prior to hospitalization at least one of the following complaints: 1) increased shortness of breath; 2) significant increase in sputum production; 3) new expectoration of purulent sputum or increased sputum purulence.

The control population included 100 post-trauma patients hospitalized in the same study period in the Orthopaedic Surgery Dept of the same medical centre who, on direct questioning, denied a febrile illness or symptoms of respiratory tract infection over the preceding month and during the interval between the drawing of two blood samples for serological testing. The study was approved by the Helsinki Committee for research on human beings of the Soroka Medical Centre, and all participants gave informed consent.

\section{Study protocol}

All patients with AECOPD were hospitalized by decision of the emergency room physicians, without intervention by the investigators. Every $24-48 \mathrm{~h}$ a research assistant visited each of the internal medicine and intensive care wards and identified patients hospitalized in the interim who met the inclusion criteria for the study. After patients agreed to participate in the study they were interviewed about their respiratory disease and it's regular treatment, their smoking habits, and symptoms of the current acute episode. During the first meeting a blood sample of $5 \mathrm{~mL}$ was drawn for serological testing. The blood was centrifuged shortly after being drawn and the serum was frozen at a temperature of $-20^{\circ} \mathrm{C}$ until serological tests were conducted. Additional relevant medical and administrative data were collected from the medical records.
Upon discharge from hospital, the patient was invited to a follow-up appointment at the pulmonary clinic of the Soroka Medical Centre, 3-5 weeks after admission to the hospital. At that clinic follow-up data were collected on the course of the convalescence and abnormal events that may have occurred following discharge from hospital. At this meeting an arterial blood sample was taken at ambient pressure, and spirometry was performed. Each patient also had a second (convalescence phase) serum sample taken for serological testing. This sample was handled in exactly the same manner as the previous acute phase sample. Patients whose hospitalization period extended for $>3$ weeks, or who were readmitted at the time of scheduled follow-up appointments, had the serum sample drawn during the hospitalization. Patients who were not considered to be in a stable condition at the first follow-up appointment (3-5 weeks after admission) were invited to an additional follow-up appointment with spirometric and arterial blood gas testing, a month later.

The study protocol for the control group included identification of patients within $48 \mathrm{~h}$ of hospitalization in the Orthopaedic Surgery Dept. After determination of their suitability for the study and obtaining of informed consent, demographical and clinical data were obtained and a blood sample was drawn for serological testing for Legionella spp. Three to 5 weeks after the first blood sample was obtained, and after any febrile illness and/or symptoms of respiratory tract infection since the first blood test were ruled out by direct questioning, a repeat serum sample was taken. The two sera were treated in the identical manner to those drawn from the study population.

\section{Serological test for Legionella spp. and other agents}

The aetiological work-up in this study was based exclusively on serological testing. Serological tests were conducted to identify 12 pathogens known to be infectious agents in the upper and lower respiratory tract, that could be diagnosed by serological methods. The paired sera for each patient were tested in the same run in all cases. The methods, kits and criteria used for serological diagnoses have been described in detail in a previous publication [5]. Antibodies to 41 different serogroups of Legionella were detected by indirect immunofluorescence using an in-house kit. Heat-killed Legionellae served as the antigen (in 17 pools). Legionella spp. were considered to be a cause of the AECOPD in the presence of a four-fold or greater increase in immunoglobulin (Ig)-G and/or IgM titre between paired serum samples.

Serological tests for seven respiratory tract viruses, Mycoplasma pneumoniae, Streptococcus pneumoniae, Haemophilus influenzae, and Moraxella catarrhalis were conducted using the enzyme immunoassay method. Only a significant change in the antibody level for a specific pathogen between the acute and convalescent serum samples was considered diagnostic for infection with that pathogen. In light of this 
requirement, only patients for whom paired sera were obtained were included in the final data analyses.

\section{Data analysis}

The Chi-squared test or it's equivalent served to compare proportions between groups, and analysis of variance (ANOVA) was inducted to compare continuous variables among two or more groups. Statistical significance was set at $\mathrm{p}<0.05$.

\section{Results}

Two hundred and forty hospital admissions for AECOPD in 213 different COPD patients during the 16.5-month period were included in the final data analyses of the study. In all admissions two serum samples were drawn at a mean interval of $24.7 \pm$ 5.6 days (range: $17-53$ days). The control population included 100 different patients with a mean age of $47.3 \pm 20.9 \mathrm{yrs}$, of whom 55 were males and 32 were current smokers. Two serum samples were taken from this group at an interval of $30.2 \pm 8.5$ days (range: $17-55$ days).

In 40 of the 240 admissions (16.7\%), a significant change of at least four-fold was observed for IgG and/ or IgM antibody titres, between the serum sample taken in the acute phase and that taken during the convalescence phase, for at least one of the 41 Lsp serogroups tested. In 11 other admissions an initially high titre was found ( $\operatorname{IgG} \geqslant 512$ and/or $\operatorname{IgM} \geqslant 64$ ), but these titres in the specific antibody did not change between the acute and convalescent sera. These 11 admissions were not considered, for the purposes of this study, to be serologically positive for Lsp.

Table 1 presents the frequency distribution for the Lsp identified in the 40 episodes. In nine of the admissions there was a significant change in antibody titre for $>1$ Lsp serogroup. The authors could not determine, on the basis of the serological tests, if this result represented a concurrent infection with $>1$ Lsp serogroup or a state of cross-reactivity among the various Lsp antibodies. Six of the 40 admissions represented pairs of admissions of three patients who were hospitalized twice each during the study period, with a time interval of $>6$ months between episodes. Details of the Lsp identified in these patients for each of the two admissions are shown in table 2. In 26 of the $40 \mathrm{LspH}(65 \%)$, serological tests identified infection with at least one other respiratory pathogen besides Lsp. In 19 cases one other pathogen was found, in two cases two other pathogens, in four cases three others, and in one case four others were identified. The frequency distribution of these other pathogens found in addition to Lsp are presented in table 3.

Among the 100 patients in the control group, only three patients had a significant change in antibody titre for Lsp. The serogroups identified in these three patients were L. pneumophila $10, L$. pneumophila 14 , and $L$. nautarum.

Table 4 shows demographical and smoking data,
Table 1. - Frequency of the various Legionella spp. identified in the 40 hospital admissions

\begin{tabular}{ll}
\hline Legionella spp. & $\mathrm{n}(\%)$ \\
\hline L. pneumophila 1 & $9(22)$ \\
L. pneumophila 3 & $3(7)$ \\
L. pneumophila 4 & $4(10)$ \\
L. pneumophila 6 & $3(7)$ \\
L. pneumophila 8 & $3(7)$ \\
L. pneumophila 9 & $1(2)$ \\
L. pneumophila 12 & $4(10)$ \\
L. pneumophila 15 & $1(2)$ \\
L. erythra & $1(2)$ \\
L. feleii & $3(7)$ \\
L. nautarum & $3(7)$ \\
L. bozemanii & $1(2)$ \\
L. gormanii & $4(10)$ \\
L. micdadei & $3(7)$ \\
L. jordanis & $4(10)$ \\
L. oakridgenesis & $1(2)$ \\
L. gratiana & $1(2)$ \\
L. londinensis & $1(2)$ \\
Seropositivity for 2 Legionella spp. ${ }^{\#}$ & $8(20)$ \\
Seropositivity for 3 Legionella spp. & $1(2)$ \\
\hline H. Ad &
\end{tabular}

\#: Admissions in which a significant antibody titre change to $>1$ species of Legionella spp. was identified.

Table 2. - Legionella spp. identified in three patients with positive serological tests in each of two admissions

\begin{tabular}{lcl}
\hline Patient & $\begin{array}{c}\text { Days interval between } \\
\text { admissions }\end{array}$ & \multicolumn{1}{c}{ Legionella spp. } \\
\hline 1 & 245 & $\begin{array}{l}\text { L. pneumophila } 6+12 \\
\text { L. pneumophila } 1\end{array}$ \\
2 & 358 & $\begin{array}{l}\text { L. bozemanii } \\
\text { L. pneumophila } 9 \\
3\end{array}$ \\
& 194 & $\begin{array}{l}\text { L. pneumophila } 1 \\
\text { L. jordanis }\end{array}$ \\
\hline
\end{tabular}

Table 3. - Frequency of pathogens identified in addition to Legionella spp. in the 40 admissions

\begin{tabular}{lc}
\hline Pathogen & $\mathrm{n}(\%)$ \\
\hline Viral agents & \\
Influenza virus type A & $1(2)$ \\
Influenza virus type B & $2(5)$ \\
Parainfluenza virus type 1 & $2(5)$ \\
Parainfluenza virus type 2 & $9(22)$ \\
Parainfluenza virus type 3 & $1(2)$ \\
Adenovirus & $1(2)$ \\
Respiratory syncytial virus & $2(5)$ \\
Bacterial agents & $12(30)$ \\
Streptococcus pneumoniae & $4(10)$ \\
Haemophilus influenzae & $2(5)$ \\
Moraxella catarrhalis & $3(7)$ \\
Atypical bacterial agents & \\
Mycoplasma pneumoniae &
\end{tabular}

results of spirometry and arterial blood gas tests in the stable condition, and information on chronic steroid therapy and rates of polycythaemia among the $40 \mathrm{LspH}$ compared to the $200 \mathrm{NLspH}$. The only significant differences between the two study 
Table 4.-Comparison of demographical data, smoking, spirometry and arterial blood gases in stable condition, steroid therapy and polycythaemia between admissions with $(\mathrm{LspH})$ and without (NLspH) serological evidence of infection with Legionella spp.

\begin{tabular}{|c|c|c|c|}
\hline Variable & LspH & NLspH & $\begin{array}{c}\mathrm{p} \text {-value } \\
\text { between } \\
\text { groups }\end{array}$ \\
\hline Subjects $n$ & 40 & 200 & \\
\hline Sex M & $36(90)$ & $166(83)$ & NS \\
\hline Mean age yrs & $64.6 \pm 8.6$ & $67.6 \pm 8.8$ & $*$ \\
\hline \multicolumn{4}{|l|}{ Smoking } \\
\hline Pack-yrs & $65.3 \pm 39.3$ & $57.6 \pm 30.0$ & NS \\
\hline Current smoker & $18(45)$ & $74(37)$ & NS \\
\hline \multicolumn{4}{|l|}{ Spirometry } \\
\hline Mean FEV1 L & $1.01 \pm 0.44$ & $1.02 \pm 0.45$ & NS \\
\hline FEV $1 \%$ of expected & $38.7 \pm 16.1$ & $41.2 \pm 16.6$ & NS \\
\hline FEV $1>50 \%$ & $9(23)$ & $53(27)$ & NS \\
\hline $35 \%<\mathrm{FEV}_{1} \leqslant 50 \%$ & $11(28)$ & $46(23)$ & NS \\
\hline $\mathrm{FEV}_{1} \leqslant 35 \%$ & $20(50)$ & $101(51)$ & NS \\
\hline FEV1/FVC & $0.565 \pm 0.120$ & $0.588 \pm 0.114$ & NS \\
\hline$P \mathrm{a}, \mathrm{O}_{2} \mathrm{mmHg}$ & $63.7 \pm 13.3$ & $68.4 \pm 12.8$ & $<0.04$ \\
\hline $\mathrm{Pa}, \mathrm{CO}_{2} \mathrm{mmHg}$ & $47.8 \pm 10.5$ & $44.4 \pm 10.2$ & NS \\
\hline \multicolumn{4}{|l|}{ Chronic steroid therapy } \\
\hline Inhaled & $15(37)$ & $48(24)$ & NS \\
\hline Oral & $14(35)$ & $56(28)$ & NS \\
\hline $\begin{array}{l}\text { Polycythaemia } \\
\text { (haematocrit }>50 \% \text { ) }\end{array}$ & $10(25)$ & $22(11)$ & $<0.03$ \\
\hline
\end{tabular}

Data are presented as value \pm SD or value $(\%)$ unless otherwise stated. M: male; FEV1: forced expiratory volume in one second; FVC: forced vital capacity; $P \mathrm{a}_{2} \mathrm{O}_{2}$ : arterial oxygen tension; $\mathrm{Pa}_{\mathrm{a}} \mathrm{CO}_{2}$ : arterial carbon dioxide tension; NS: nonsignificant. ${ }^{*}: \mathrm{p}<0.05$.

populations were the younger mean age, and higher rates of hypoxaemia and polycythaemia in the $\mathrm{LspH}$ group.

Table 5. - Comparison of clinical features of exacerbations with $(\mathrm{LspH})$ and without $(\mathrm{NLspH})$ serological evidence of infection with Legionella spp.

\begin{tabular}{lccc}
\hline Variable & LspH & NLspH & $\begin{array}{c}\text { p-value } \\
\text { between } \\
\text { groups }\end{array}$ \\
\hline $\begin{array}{l}\text { Subjects n } \\
\text { Increased dyspnoea (days }\end{array}$ & 40 & 200 & \\
$\quad$ before hospitalization) & $6.6 \pm 3.3$ & $5.0 \pm 3.6$ & $* *$ \\
Exacerbation & $24(60)$ & $101(50)$ & NS \\
$\quad$ Type 1 & $5(12)$ & $36(18)$ & NS \\
$\quad$ Type 2 & $11(28)$ & $63(32)$ & NS \\
$\quad$ Type 3 & $0(0)$ & $58(29)$ & $\#$ \\
Abrupt onset & $3(8)$ & $12(6)$ & NS \\
$\quad$ of exacerbation & $24(60)$ & $100(50)$ & NS \\
Confusion & $22(55)$ & $74(37)$ & $<0.03$ \\
Headache & $6(15)$ & $24(12)$ & NS \\
Myalgia/arthralgia & $14(35)$ & $50(25)$ & NS \\
Nausea/vomiting & $4(10)$ & $12(6)$ & NS \\
Abdominal pain & $27(68)$ & $142(71)$ & NS \\
Diarrhoea & Fever (at any & & \\
$\quad$ stage of disease) & & & \\
\hline
\end{tabular}

Data are presented as value \pm SD or value (\%) unless otherwise stated. ": in accordance with the classification of ANTHONISEN et al. [6]; **: $\mathrm{p}<0.01$; ${ }^{\#}: \mathrm{p}<0.0001$.
Table 5 presents a comparison of clinical expressions of AE between the LspH and NLspH groups. There was no difference between the study groups in the clinical type of exacerbation using the classification proposed by ANTHONISEN et al. [6]. A significant and striking difference was found in the rate of abrupt onset of the exacerbation. While none of the patients in the LspH group had an abrupt onset of AE, $29 \%$ of the NLspH did, with onset occurring within $12 \mathrm{~h}$. The time interval between the onset of AE to hospitalization was significantly longer in the $\mathrm{LspH}$ groups than in the NLspH group. Comparison of the systemic (extra-pulmonary) manifestations that accompanied the exacerbation in the $\mathrm{LspH}$ and NLspH groups shows that all of these features were more apparent in the LspH group although the difference reached statistical significance only in the case of myalgia/ arthralgia.

The study database includes a broad range of additional data on the hospital admissions included in the study. Comparisons of the $\mathrm{LspH}$ and $\mathrm{NLspH}$ groups for these variables showed no other statistically significant differences between the groups. Of interest, among these comparison variables are the number of admissions in the year preceding the index hospitalization, immunization rates for influenza and pneumococcus, type and rate of chronic comorbidity, type and frequency of antibiotic therapy prior to, during and after hospitalization, vital signs and findings on admission physical examination, results of tests for arterial blood gases and routine biochemistry tests on admission, rate of admission to intensive care, mechanical ventilation and mortality, duration of hospitalization and number of readmissions, days of inactivity following hospitalization and time to return to normal function.

Of the $40 \mathrm{LspH}$ there were three admissions (8\%) to intensive care (versus $9 \%$ in the $\mathrm{NLspH}$ ) and six admissions (15\%) in which the patients required respiratory assistance (versus $9 \%$ in the $\mathrm{NLspH}$ ), including two with invasive mechanical ventilation and four with noninvasive ventilation. Two patients $(5 \%)$ in the $\mathrm{LspH}$ group died (versus $2 \%$ in the $\mathrm{NLspH}$ ). One was a 54-yr-old male with serological evidence of infection with $L$. erythra and influenza $B$ virus, who died after 21 days of mechanical ventilation. The other was a 70 -yr-old male with serological evidence of infections with L. erythra, L. pneumophila 12 , and $L$. nautarum, who died after 35 days of mechanical ventilation. These two patients were treated with a broad range of antibiotics in the course of their admission, including macrolides and fluoroquinolones.

\section{Discussion}

The major, original finding of this study was the relatively high rate of $17 \%$ of hospital admissions for AECOPD with serological evidence of acute infection with Lsp that occurred around the time of the episode. This rate represents a significant difference from previous publications in which Lsp is completely absent from the list of pathogens that cause 
AECOPD. This important finding is attributed to two methodological advantages of the present study. The first is the use of a sensitive diagnostic test for the identification of 41 different serogroups of Lsp. The second is the availability of acute and convalescent sera for each of the admissions.

In terms of diagnosis there are two points worthy of discussion. First, the diagnosis of Lsp infection in this study was based solely on serological measurements and not on identification or isolation of the pathogen in respiratory secretions. The reason for this preference lies in the combination of the technical difficulty involved in identifying or isolating all the various Lsp serogroups in cultures, together with the possibility that isolation of pathogens from secretions may reflect a carrier state and not necessarily a state of infection. The authors believe that these two reasons account for the fact that in routine clinical work, as well as in the vast majority of studies dealing with infections caused by all serogroups of Lsp, the accepted diagnostic method is serology and not identification or isolation from cultures. It was not practicable to diagnose infection using the urinary antigen of Legionella in this study, because the commercial urinary kits available today only identify the antigen of $L$. pneumophila 1 , which was found in only $22 \%$ of $\mathrm{LspH}$ in the present study.

The serological tests for Legionella spp. in this study were conducted using an in-house kit and it is important to note that there is no commercially available kit with which it is possible to test for all the 41 serogroups of Legionella spp. that were tested in the current study. Although use of an in-house kit is problematic, this is a universal problem and not specific to the present study. The problem stems, primarily, from the absence of a gold standard against which results of serological tests can be validated. Cultures of pathogens, as well as polymerase chain reaction detection, have low sensitivity and cannot be used as a gold standard. The accepted substitute for validation of test results is strict quality controlled/ quality assured procedures together with rigid diagnostic criteria, using paired sera. Both of these conditions were strictly adhered to in this study.

The second point relating to diagnosis is the diagnostic criteria used in this study for the determination of acute Lsp infection. Although high single titres above a predetermined threshold value of IgG and/or $\operatorname{IgM}$ are usually accepted as diagnostic for acute infection, the authors did not adopt this approach as in all 11 admissions in which this criterion alone was fulfilled, the high titres in the specific antibody did not change between the acute and convalescent sera. This undermined the confidence that in these admissions there was an acute Lsp infection, and raised the possibility that the high titre was the result of past infection. In light of this, acute Lsp infection was diagnosed only in situations in which there was a significant difference in antibody titre in one of these two types of antibodies. It is possible that this decision decreased the sensitivity of the serological diagnosis in this study, but on the other hand, it increased the specificity.

The possibility that the changes in antibody titre to
Lsp were coincidental and could have been found without association with AECOPD was tested, by using a control group of patients without clinical evidence of an acute respiratory tract infection. Although the control group was not matched to the study group, the authors believe that it is suited to answer this specific question. Using identical diagnostic methods and criteria, significant changes in antibody titre to Lsp in only $3 \%$ of the control patients was found, a difference which is an order of magnitude more than the rate found in the population of patients hospitalized with COPD. It is assumed that the three positive controls cases had subclinical exposure to a specific serogroup of Lsp. This rate of $3 \%$ should be viewed as "background noise" of the test. In addition, three patients with repeat admissions had positive serological tests for Lsp in each of the two exacerbations, but with a different Lsp serogroup on each occasion. These results, together with the unique clinical characteristics found for $\mathrm{LspH}$ represent, in the authors opinion, proof of a true association between Lsp infection and the episodes of AECOPD in which it was identified.

An additional interesting and important finding in the present study was that in $65 \%$ of $\mathrm{LspH}$ at least one additional respiratory pathogen was found associated with AE. In most of these cases only one additional pathogen was found, but in seven LspH 2-4 other pathogens were identified. This situation of "mixed causes" in infective respiratory tract diseases is well known and has been reported in a large number of studies on the infective aetiology of CAP [1], as well as in a study that investigated the causes of respiratory tract infections in adults in a general practice setting [7]. Although mixed causes have been described for all possible combinations of respiratory tract pathogens, the frequency of a combined aetiology is particularly high when one of the causes is an atypical pathogen [1]. The rate of $65 \%$ in this study is almost identical to the $63 \%$ of CAP patients with serological evidence of Lsp infection in which an additional pathogen was identified [8]. In light of this finding it is important to discuss the issue of the status of Lsp in $\mathrm{LspH}$ patients with an additional pathogen. Did the Lsp infection in these patients cause or contribute to the clinical manifestations of the disease, or perhaps it only facilitated penetration of the additional infection that was then responsible, in turn, for the clinical expression of infection? The serological findings, per se, cannot provide an answer to this question. The significant change in antibody titre can be an expression of a previous infection, a simultaneous infection, or an infection that follows another in time. Analysis of clinical findings also cannot provide a convincing answer to this question. The fact that there is no difference in outcome in relation to specific antibiotic therapy may provide a clue that at least in some of the patients the contribution of Lsp to the exacerbation was not cardinal.

Although no difference was found in the clinical type of the AE, in accordance with the classification of ANTHONisen et al. [6], between LspH and NLspH, several significantly different clinical characteristics were seen between the two groups. The most striking 
difference was the sudden onset of the exacerbation seen in $29 \%$ of the NLspH group, but not in a single case of LspH. Another difference was the higher rate of systemic manifestations in $\mathrm{LspH}$ that reached statistical significance for myalgia/arthralgia. This difference is consistent with the known systemic nature of infection with Lsp. These two clinical differences between the groups supports the conclusion that the serological findings also have a clinical relevance.

The authors conclude that serological evidence for Legionella spp. infection is common in patients hospitalized for acute exacerbations of chronic obstructive pulmonary disease and that in most of these patients an additional infective respiratory pathogen can be identified. The clinical expression of exacerbations in these patients is characterized by gradual onset and more systemic manifestations. The true interpretation and practical implications of these findings should be tested in further studies.

\section{References}

1. Lieberman D. Atypical pathogens in communityacquired pneumonia. Clin Chest Med 1999; 20: 489-497.
2. Griffith DE, Mazurek GH. Pneumonia in chronic obstructive lung disease. Infect Dis Clin North Am 1991; 5: 467-484.

3. Torres A, Dorca J, Zalacain R, et al. Communityacquired pneumonia in chronic obstructive pulmonary disease: a Spanish multicenter study. Am J Respir Crit Care Med 1996; 154: 1456-1461.

4. Goh SK, Johan A, Cheong TH, Wang YT. A prospective study of infections with atypical pneumonia organisms in acute exacerbations of chronic bronchitis. Ann Acad Med Singapore 1999; 28: 476-480.

5. Lieberman D, Lieberman D, Ben-Yaakov $\mathrm{M}$, et al. Infectious etiologies in acute exacerbation of COPD. Diagn Microbiol Infect Dis 2001; 40: 95-102.

6. Anthonisen NR, Manfreda J, Warren CPW, Hershfeld ES, Harding GKM, Nelson NA. Antibiotic therapy in exacerbations of chronic obstructive pulmonary disease. Ann Intern Med 1987; 106: 196-204.

7. Lieberman D, Shvartzman $\mathrm{P}$, Lieberman $\mathrm{D}$, et al. Etiology of respiratory tract infection in adults in a general practice setting. Eur J Clin Microbiol Infect Dis 1998; 17: 685-689.

8. Lieberman D, Porath A, Schlaeffer F, Lieberman D, Boldur I. Legionella species community-acquired pneumonia: a review of 56 hospitalized adult patients. Chest 1995; 109: 1243-1249. 